\title{
Idiosynkrasie und Systematik in KünstlerInnenarchiven
}

\author{
Fallbeispiel: VALIE EXPORT Center Linz
}

Dinge in der Vergangenheit, die aber Gegenwart haben. (Valie Export)

\section{Das Ein-Personen-Archiv als Vorlass}

VALIE EXPORT hat sich als Pionierin der Medien- und Performancekunst intensiv mit ihrer politischen Gegenwart auseinandergesetzt. So ist ihr Werk über einen ästhetisch-künstlerischen Forschungsanspruch hinaus als eine mit Nachdruck und Leidenschaft betriebene anthropologische Unternehmung zu begreifen: Die Analyse der Mechanismen von Macht, Disziplinierung, Kontrolle sowie der Wechsel der Paradigmen durch neue Technologien und die damit verbundenen Implikationen für das Subjekt stehen im Mittelpunkt. Das weibliche Subjekt und dessen Zurichtung sind dabei vordringlich interessant, aber grundsätzlich geht es um die Auswirkungen einer Spaltung des gesellschaftlichen Bewusstseins in weiblich/männlich, Natur/Kultur, Subjekt/Objekt - Dichotomien, die paradoxerweise gerade durch das Projekt der Aufklärung mit ihrer Hypostasierung der Vernunft befördert wurden. Andererseits verschreibt sich EXPORTs Denken seit Beginn an dem impliziten oder expliziten Abarbeiten der Traumata des 20. Jahrhunderts - der Kriege, des Holocaust und der damit verbundenen Wiederkehr des Verdrängten - sowie den Bedingungen von Ausbeutung, Gewalt, globalem Kapitalismus und schließlich der Verführung durch das Prothetische im digitalen Zeitalter samt seiner Vorgeschichte vor allem im 19. Jahrhundert.

Die Notwendigkeit, auf der einen Seite Äußerungsformen wie Aktionen, Performances und schwer einzuordnende konzeptuelle, mediale, ständig auf ein „Dazwischen“ verweisende Experimente festzuhalten sowie ausfransende, überlappende, im Technischen und Technologischen gründende Realitätsebenen zu erkunden, die Bereiche der Physik, Verhaltensforschung, der Biologie, der Sozialforschung und viele andere Felder tangieren; und auf der anderen Seite der Wille, dem, was angegriffen, ausgelöscht werden kann, in seiner Natur nach ephemer ist, dem Prozess des Verschwindens vorzuenthalten: Dies dürften nur einige der Beweggründe sein, die die Künstlerin dazu veranlasst haben, zu sammeln und zu dokumentieren und in weiterer Folge zu ,archivieren“. Im Archivieren selbst ist 
zweifellos eine weitere Ebene der Reflexion, der Bewertung und Sondierung angelegt, die über den reinen Werkcharakter eines abgeschlossenen Werks hinausweisende Aspekte beinhaltet.

Wenn man so will, ist EXPORTs Arbeit aber auch selbst ein Geschichtsarchiv, das Gegenmodell existierender Archive, der Versuch, Geschichte neu zu schreiben, das hegemoniale Herrschaftsgefüge $z u$ destabilisieren, das Archiv der Geschichte zu dekonstruieren von festgefügten Identitätskonstruktionen, es zu dekolonisieren. Ihr Archiv erzählt daher auch die Geschichte eines bürokratischen Geschehens, der Apparate, die im Hintergrund wirken, und es gibt andererseits Auskunft darüber, wie künstlerische Prozesse funktionieren, wie Einflüsse, gefundenes Material, Literatur, technologische Entwicklungen auf Äußerungsformen einwirken.

Neben dem Fakt, dass VALIE EXPORT immer schon daran gelegen war, ihre Denk- und Entwicklungsprozesse zu archivieren oder zumindest zu dokumentieren, orientierte sich ihr Bestreben zunehmend dahin, ihr akribisch zusammengetragenes Material einer interessierten Öffentlichkeit zugänglich zu machen und als Ausgangspunkt zur Erforschung der (feministischen) Performance- und Medienkunst zur Disposition zu stellen - bezogen auf ihr Werk, aber auch auf diese Felder allgemein.

Ihre dahingehenden rund sieben Jahre währenden Bemühungen, Überzeugungsarbeit für die Errichtung eines international einmaligen Ein-PersonenArchivs zu leisten, es an eine oder mehrere Institutionen anzubinden, waren schließlich erfolgreich: 2015 hat die Stadt Linz den Vorlass der in Linz geborenen Künstlerin gekauft und dem LENTOS Kunstmuseum überantwortet. In Kooperation mit der Kunstuniversität Linz betrieben, ist in Folge im von Peter Behrens und Alexander Popp geplanten historischen Bau der Tabakfabrik aus den 1930erJahren im November 2017 das VALIE EXPORT Center Linz in eigenen Räumlichkeiten eröffnet worden - als Archiv und als Forschungszentrum für Medien- und Performancekunst. Die Stadt kommt für die Infrastruktur auf, die Universität für Personal und Programm, und zusätzliche Drittmittel für u. a. Digitalisierung, Sonder- und Forschungsprojekte müssen beschafft werden.

Seit der Eröffnung hat es neben der Einrichtung der Infrastruktur, den diversen Kollaborationen, der fortlaufenden Digitalisierung und dem allgemeinen Betrieb/Programm einige Präsentationen des Vorlasses gegeben, denen Überlegungen aus kuratorischer Perspektive zugrundeliegen, wie mit der Visualisierung eines Archivs, das sich tendenziell einer musealen Präsentation widersetzt, im Format der Ausstellung umgegangen werden kann. Dazu fanden bislang drei von mir kuratierte Ausstellungen mit wechselnden Varianten von Displays und Bezugsebenen zwischen Werk und Archiv statt: im LENTOS Kunstmuseum (2017/2018), im Neuen Berliner Kunstverein (2018) und im VOX Centre de l'image 
contemporaine in Montreal (2019). Eine weitere, von Dagmar Schink kuratierte Ausstellung wurde am IFK in Wien ausgerichtet (2018). Wir betrachten es als integralen Bestandteil unserer Arbeit, das Archiv nicht nur zu beforschen und über Publikationen zugänglich zu machen, sondern auch über das Format der Ausstellung aufzuarbeiten, zu „zeigen“, zu kontextualisieren, Verweise und Bezüge auch visuell herzustellen. Das Eingehen auf diese Visualisierungsformen in Ausstellungen über Diagramme, Grafiken, abstrahierte Übertragungen von faktischen Objekten und Dokumenten bildet einen zentralen Fokus meiner Arbeit, ist aber hier nicht Gegenstand der Auseinandersetzung.

Der Vorteil eines erfassten, systematisierten und dann beforschten Vorlasses gegenüber einem Nachlass ist, dass offene Fragen an die noch lebende „Bestandsbildnerin“ möglich sind und ihren wertvollen Informationen nachgegangen werden kann. Hinweise in Bezug auf Transkribierungen, Übersetzungsvorgänge im eigentlichen und übertragenen Sinne zur hermeneutischen Erschließung des Werks können gegeben werden. Andererseits wird der Künstlerin selbst die Möglichkeit gewährt, mitzuerleben, wie mit Formen des Re-enactments umgegangen wird oder wie Teile ihres Werks zum Ausgangspunkt für neue Werke von jungen KünstlerInnen werden oder auch wie durch Fellows, ForscherInnen neue Forschungsaspekte zutage treten.

Ziel ist es, den Vorlass über die Beforschung anschlussfähig und fruchtbar für gegenwärtige Diskurse zu machen, die vielfach bereits vor einem halben Jahrhundert durch EXPORTs künstlerische Forschung angestoßen wurden.

\section{Logiken der Sammlung von Künstlerarchiven}

Jürgen Thaler hat in seinem Text zur Ausstellung VALIE EXPORT - ARCHIV am Kunsthaus Bregenz 2011/12 die hilfreiche Unterscheidung vom „naiven“ Archiv (nach Yerushalmi und in weiterer Folge von Derrida) und dem „sentimentalischen“ Archiv (nach Schiller) aufgegriffen, um Unterscheidungsmöglichkeiten von Staatsarchiven und individuellen Archiven zu bezeichnen (vgl. Thaler 2012): Demnach ist das „sentimentalische“ Archiv persönlich, anarchisch, folgt einer idiosynkratischen Ordnung und ist keiner rechtsstaatlichen Ordnung unterworfen. Man könnte auch mit Walter Benjamin sagen, Künstler sammeln wie „Lumpensammler“: Sie sammeln gegen das Herrschaftsverständnis des dominierenden Diskurses, nach einer eigenen Ordnung, die unintentional/intentional/ intuitiv/subjektiv/selektiv ist, eher einem Sammeln nach Ähnlichkeiten, Analogien, Sympathien verpflichtet. Damit ergibt sich der Vergleich zwischen dem Künstler/der Künstlerin und der Indifferenz des Kindes beispielsweise gegenüber dem common sense (vgl. Finkelde 2006) des Kanons, einer Nomenklatur des Sam- 
melns, das heißt es handelt sich um das Erstellen einer Ordnung jenseits der symbolischen patriarchalen Ordnung.

In diesem Zusammenhang stehen zunächst einige Aussagen der Künstlerin selbst zum Archiv:

Sicherlich ist es hauptsächlich mit dem künstlerischen Tun entstanden, aber soweit ich mich erinnern kann, habe ich schon als Kind sehr gerne gesammelt (muss man in dem Fall sagen) - es war eine Anhäufung von Dingen, die einerseits meine Vergangenheit und die Gegenwart waren, aber wo ich auch wusste, es hat auch etwas Zukünftiges. Als Kind weiß man es nie so dezidiert und kann es vielleicht auch gar nicht genau auseinanderhalten, weshalb man sammelt. Zum Beispiel habe ich aus Fotos meines Vaters, der im Krieg gefallen ist und an den ich mich nicht mehr erinnern konnte und anderen Fotos, Altäre gebaut. Die Fotos von meinem Vater waren seine Lebenslinien, bei denen es für mich aber damals vollkommen normal war, dass sie JETZT sind. Es war natürlich nicht JETZT, es war meine Fantasie. Es war ein Spiel, um ihn mehr kennenzulernen. Ein Archiv funktioniert letzten Endes auch in dieser Art, dass man in das Archiv hineingreift, um etwas herauszuziehen; man will etwas Unbekanntes kennenlernen oder einen Zusammenhang finden, wenn man wieder etwas anderes herauszieht. (Zit. n. Winkelmayer 2016, 5)

So wie es VALIE EXPORT mit den Erinnerungsstücken ihres Vaters ergangen ist, dieser Wille zur Rekonstruktion, ergeht es uns und den ForscherInnen generell, wenn wir das Material in die Hände bekommen und versuchen, ein Puzzle zusammenzufügen, zu vergleichen, abzuleiten, Schlüsse zu ziehen. Zur Differenz aber der Sammlung eines Museums im Vergleich zur Akkumulation von Dokumenten in einem Archiv wie dem ihren, meint EXPORT:

Eine Sammlung in einem Museum ist ein öffentliches Gut, ist ein öffentliches Gedächtnis, ein Museumsgedächtnis, die Zugänglichkeit ist künstlich gemacht. Sie könnte ja auch offen sein. Ein Archiv und eine Sammlung haben auch konträre Strukturen, wenn sie mit Gedächtnis zu tun haben. Innerhalb eines Archivs gibt es viel mehr Verbindungen und Wege als in einer Sammlung, was die Dinge zusammenbringt oder zusammenhält. Eine Sammlung kann auch sehr einförmig sein, obwohl sie natürlich verschachtelt, verschiedenen Ursprungs und Kontextes ist. Man könnte auch sagen, ein Archiv hat etwas - wenn man einen Begriff verwenden darf, der nicht mehr in unserer Zeit verwendet wird - Anarchistisches in sich -, weil es verschiedene Dinge aufgreift, zusammenbringt und nicht einer starken Regel unterliegt. Die Regeln werden gebrochen bzw. sie verteilen sich wie ein Rhizom. Die Strukturen sind anarchistischer, weil man unterschiedliche Strukturen oder Materialitäten zu Aussagen zusammenbringt - das mag eine Sammlung nicht leisten. (Zit. n. Winkelmayer 2016, 5-6)

Am anderen Ende dieses Prozesses oder auf der anderen Seite steht das hermeneutische Interesse der ForscherInnen und KuratorInnen: Material wird durchgesehen, Bezüge hergestellt zu vollendeten und unvollendeten Werken resp. unvollendeten oder nicht umgesetzten Ideen, Drehbüchern etc. Es ist unsere Aufgabe, 
einzelne Werke oder das Werk insgesamt um Erkenntnisse zu erweitern, die sich oft aus dem fertig gestellten Werk allein nicht ergeben. Andererseits erweitern die Skizzen und Konzepte, die nicht aus dem Archiv herausgetreten sind, die Wahrnehmung, stiften neue Zusammenhänge. Dabei gibt es natürlich kontinuierlich auch das Problem der blinden Flecken, Informationslücken, Fragezeichen, das VALIE EXPORT im Nachdenken über das Archiv folgendermaßen umschreibt: „Was zwischen den einzelnen ,Dingen“ ist, ist Rauschen, Effekt TV, Information in einem nichtspezifischen Frequenzspektrum wie es in der Physik ausgedrückt wird“ (zit. n. Winkelmayer 2016, 14); „Wäre auch interessant, die Themen, die Vorstellungen, Ideen etc. so zu ,untersuchen', dass sich daraus auch Beschäftigungskurven ergeben“ (zit. n. Winkelmayer 2016, 14).

\section{Idiosynkrasie und Systematik im Archiv von VALIE EXPORT}

KünstlerInnenarchive gehorchen also anderen Gesetzen, als es landläufige Archive tun. Folgende Aussage EXPORTs belegt diesen Befund:

Ich habe den Vorteil gegenüber dem Museum oder dieser Art von Sammlungen, dass ICH immer ICH bin. Im Museum sammeln ja verschiedene Identitäten - Direktor_innen, Auswahlgremien, Beirät_innen -, die dann ihre Interessen vertreten und vielleicht gar nicht so sehr den Archivbegriff in sich tragen. Das kann bei meinem Archiv nicht passieren, weil ich immer diejenige bin, die auswählt und die die Zusammenhänge schafft bzw. die Zusammenhänge darstellt, „,visuelle Strukturen“ schafft. (Zit. n. Winkelmayer 2016, 6)

Insofern kann man davon sprechen, dass diese Archive und auch VALIE EXPORTs Archiv einer idiosynkratischen Ordnung folgt, was impliziert, dass es eine eigene Ordnung ist.

Daraus folgt: Man kann von einer Logik der Sammlung der „Bestandsbildnerin“ versus einer Logik der Systematisierung des Archivs als bewahrender Entität sprechen, wobei letztere, die Systematisierung, im Zuge der Erschließung des Bestands für die Beforschung eine Notwendigkeit darstellt. Viele Schritte sind notwendig: Digitalisieren, Ablegen, Systematik erstellen und Signaturen vergeben, Findbuch erstellen, Beforschen, Zeigen (Ausstellen).

Es handelt sich in gewisser Weise um gegenläufige Logiken: Jene idiosynkratische Ordnung mit all den Eigentümlichkeiten, Auswahlverfahren, geleiteten Interessen, die aber nicht durchgängig in der Systematik der Ablage und Archivierung nachvollziehbar ist, steht der Notwendigkeit und „Logik“ gegenüber (1) Dinge zu finden/abzulegen (Zusammenhänge zu erstellen, Systematik zu erstellen) und (2) sie zu lesen, zu interpretieren, zusammenzuführen, was eventuell auseinandergerissen wurde oder aus der historischen Perspektive aufgrund 
der zwangsläufigen Dokumentationslücken nicht mehr eindeutig nachvollzogen werden kann.

Es gibt Systematiken der Bibliothek, der Dokumente, Skizzen und Videos, die vertikal und horizontal gewissermaßen teilweise nach Medien organisiert sind (Korrespondenz nach Jahren, Projekten geordnet, Videosammlung, Dias, Drucksorten, Fotos etc.), und nach Themen/Projekten/Werken. Natürlich trifft man hier auch gerade in den 1980er-Jahren auf die Typoskripte, die mit der mechanischen Schreibmaschine getippt wurden und später mit dem Computer und damit zusammenhängend auf die oftmals schwer zu benützenden Datenträger/Disketten etc. Die Prozesse der künstlerischen Arbeit werden neben Skizzen und Konzepten unter anderem über diverse Archivalien sichtbar und die Persona der Künstlerin, die Facetten der Aktivitäten, zeigen sich.

Von außen betrachtet scheint es, dass VALIE EXPORT akribisch gesammelt hat, alles aufbewahrt und kaum skartiert hat, das heißt, es gibt sehr viel Material, wie Typoskripte, die in mehrfachen Ausfertigungen und, ja, Überarbeitungen, vorhanden sind. Das heißt, die verschiedenen Stufen und Überarbeitungen von Texten beispielsweise, aber auch von Werken, sind oft belegt.

\section{Das hermeneutische Interesse}

Was mir immer am schwersten fällt in meinem Gedankenarchiv ist die Zuordnung. Ich ordne Dinge zu und merke aber, dass noch viele andere Zuordnungen möglich wären und ich müsste die Unterlagen auch dort noch zuordnen. Die Archivstruktur war von mir nicht als Archivstruktur gedacht. (Zit. n. Winkelmayer 2016, 7)

Ein Archiv ist immer in sich „verschachtelt“, wie eine Komposition, die auch immer wieder Kompositionsteile aufgreift, „verändert“ unterschiedlich zusammenfügt. Man könnte auch sagen eine mediale, filmische „Super-Überblendung“. So habe ich auch mein Archiv immer gesehen. Ich habe nicht archiviert, weil ich dachte, ich werde das JETZT für eine spätere Zeit aufheben, sondern weil es in dieser Zeit WAR und weil ich mir dachte, dass ich DIESE Zeit mittels einer Gedächtnisstruktur und dem materiellen Gedächtnis - also der Teile des Archivs - in die zukünftige Zeit transferieren kann. (Zit. n. Winkelmayer 2016, 4)

Zusammenfassend: Das Archiv ist wie jedes KünstlerInnenarchiv ein idiosynkratisches und die Aufgabe, die sich nun stellt, ist diesem idiosnykratischen Archiv mit einer gewissen „wissenschaftlichen“ Systematik zu begegnen. Es gilt, die ursprüngliche Ordnung von VALIE EXPORT zwar zu erhalten, damit gewisse Nachbarschaften, Nähen, die auf den ersten Blick nicht erkennbare Bezüge erhellen, erhalten bleiben, gleichzeitig aber das zu machen, was VALIE EXPORT mit dem Satz: „Teile werden zu einem Archiv-,Skelett‘ zusammengefügt“ beschrieben 
hat, anders ausgedrückt, für uns und die ForscherInnen analog und digital nachvollziehbare Ordnungskriterien zu schaffen, die zwischen diesen beiden Ordnungen über Konkordanzen, Findbücher etc. Dinge zueinander bringen, inhaltliche Bezüge schaffen, die in der „Logik der Sammlung“ der Künstlerin oft auf unterschiedliche Ordnungssysteme verteilt sind. Das hängt nicht zuletzt damit zusammen, dass beispielsweise für den Transport nochmals eine eigene Ordnung (Boxen, Schachteln) geschaffen werden musste.

\section{Das Verhältnis von Archiv und Werk}

Die Archivarbeit an so einer Sammlung ist eine forensische Arbeit, die durchzogen ist von Variablen, Unwägbarkeiten und Kontingenzen. Im Fall VALIE EXPORT um so mehr, als das Archiv letztlich einen Teil der künstlerischen Arbeit darstellt, diese selbst aber nicht Gegenstand des Archivs ist, d. h. das Archiv besteht aus Recherchedokumenten, Korrespondenzen, Aussortiertem, Skizzen, Büchern, Zeitungsartikeln, die mitunter zu den Werken hinführen, aber dessen Bestandteile nicht selbst Werk sind. Und ihr Archiv ist auch nicht mit dem ,archival impulse“, den Hal Foster für viele künstlerische Ansätze seit den 1960er-Jahren als maßgeblich konstatiert, zu definieren (vgl. Foster 1967): In diesen steht nicht nur der Prozess, der in Notaten, Skizzen, Entwürfen in Archiven abgebildet wird, oft gleichwertig neben dem Werk, vielmehr ist das Archiv der Bilder selbst grundsätzlich Gegenstand der künstlerischen Praxis. Oder es trifft eine andere Variante zu: Das, was gängigerweise eine Archivalie wäre, eine Konzeptskizze wird in der Konzeptkunst zum integralen Bestandteil der Kunst selbst, wenn nicht sogar der entscheidende: denn die Idee ist das Konzept und die Ausführung, wie wir wissen, nicht zwangsläufig vom Künstler/der Künstlerin selbst auszuführen. Oder wie es Sol LeWitt ausgedrückt hat: „Alle Zwischenschritte - Kritzeleien, Skizzen, Zeichnungen, gescheiterte Arbeiten, Modelle, Studien, Gedanken, Gespräche - sind von Interesse. Solche, die den Denkprozess des Künstlers zeigen, sind oft interessanter als das finale Produkt“" (LeWitt 1967, 82).

KünstlerInnenarchive als Orte künstlerischer Forschung treten zunehmend in den Fokus, sei es als Quelle zur Erforschung von Werkkomplexen oder eben als künstlerische Methode. Wie wäre also die Grenzlinie zwischen einem Archiv als Dokumentensammlung und einem Archiv, das sich über die Ästhetik und die (vermeintliche) Verweiskraft des Historischen des Archivs - des eigenen Archivs oder fremder Archive - konstituiert, zu ziehen? Und welche Definition trifft auf die Anlage des Archivs von VALIE EXPORT zu? Diese Frage ist schnell beantwortet: EXPORTs Archiv verdankt sich in erster Linie dem Überlieferungswillen der 
Bestandsbildnerin, die das eigene Werk mit dem Gedächtnisraum einer Zeit „in die zukünftige Zeit transferieren“ (zit. n. Winkelmayer 2016, 4) will.

Die Grenzziehungen sind allerdings fließend, denn: ist nur der Film das Werk und das Drehbuch nicht, welches sich im Archiv befindet und warum sind manche Skizzen Teil des Werks, andere nicht? Hier stoßen wir auf eine spezielle „Logik der Sammlung“, die nicht zuletzt davon bestimmt ist, mit welchem Werkbegriff wir es zu tun haben. Zweifellos ist dieser in ephemeren Kunstpraktiken wie der Performance und Konzeptkunst generell stets von Neuem zu definieren und nicht immer eindeutig. Und so gibt es gewiss Grauzonen, mit denen das Archiv und wir uns auseinandersetzen müssen - ein anhaltendes Abenteuer.

\section{Literaturverzeichnis}

Derrida, Jacques. Dem Archiv verschrieben. Berlin: Brinkmann + Bose, 1997.

Finkelde, Joachim. „Vergebliches Sammeln. Walter Benjamins Analyse eines Unbehagens im Fin de Siècle und der europäischen Moderne “. arcadia 41.1 (2016): 187-202.

Foster, Hal. „An Archival Impulse“. October 110 (2004): 3-22.

LeWitt, Sol. „Paragraphs on Conceptual Art“. Artforum 5/10 (1967): 79-83.

Thaler, Jürgen. „Archivalische Konstellationen: VALIE EXPORT“. VALIE EXPORT Archiv.

Hg. Yilmaz Dziewior. Köln: Verlag der Buchhandlung Walther König, 2012. 23-31.

Winkelmayer, Sylvia. Different und Synonym. Bemerkungen zum Archiv VALIE EXPORT.

Wien: Univ. Diss., 2016. 

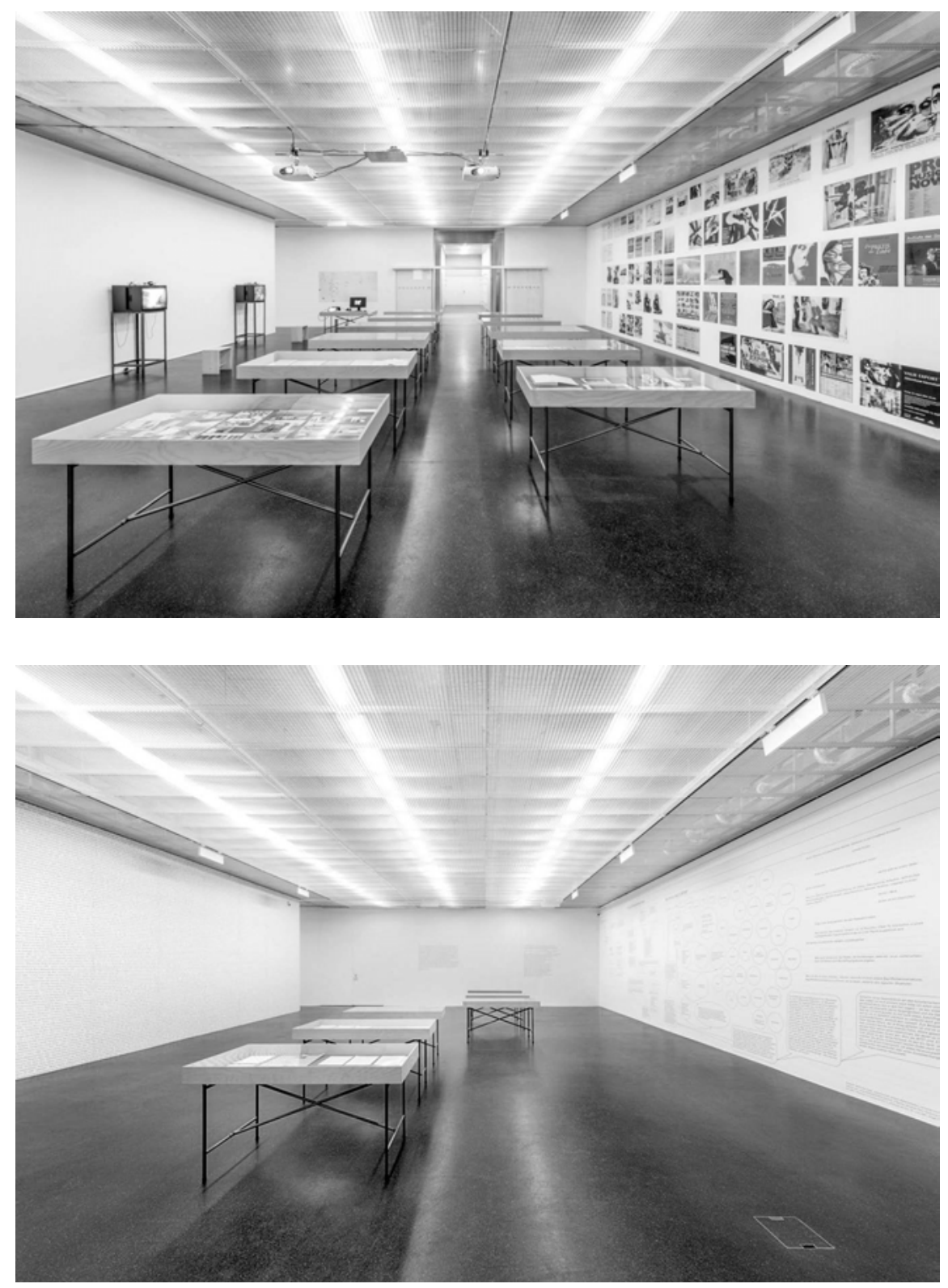

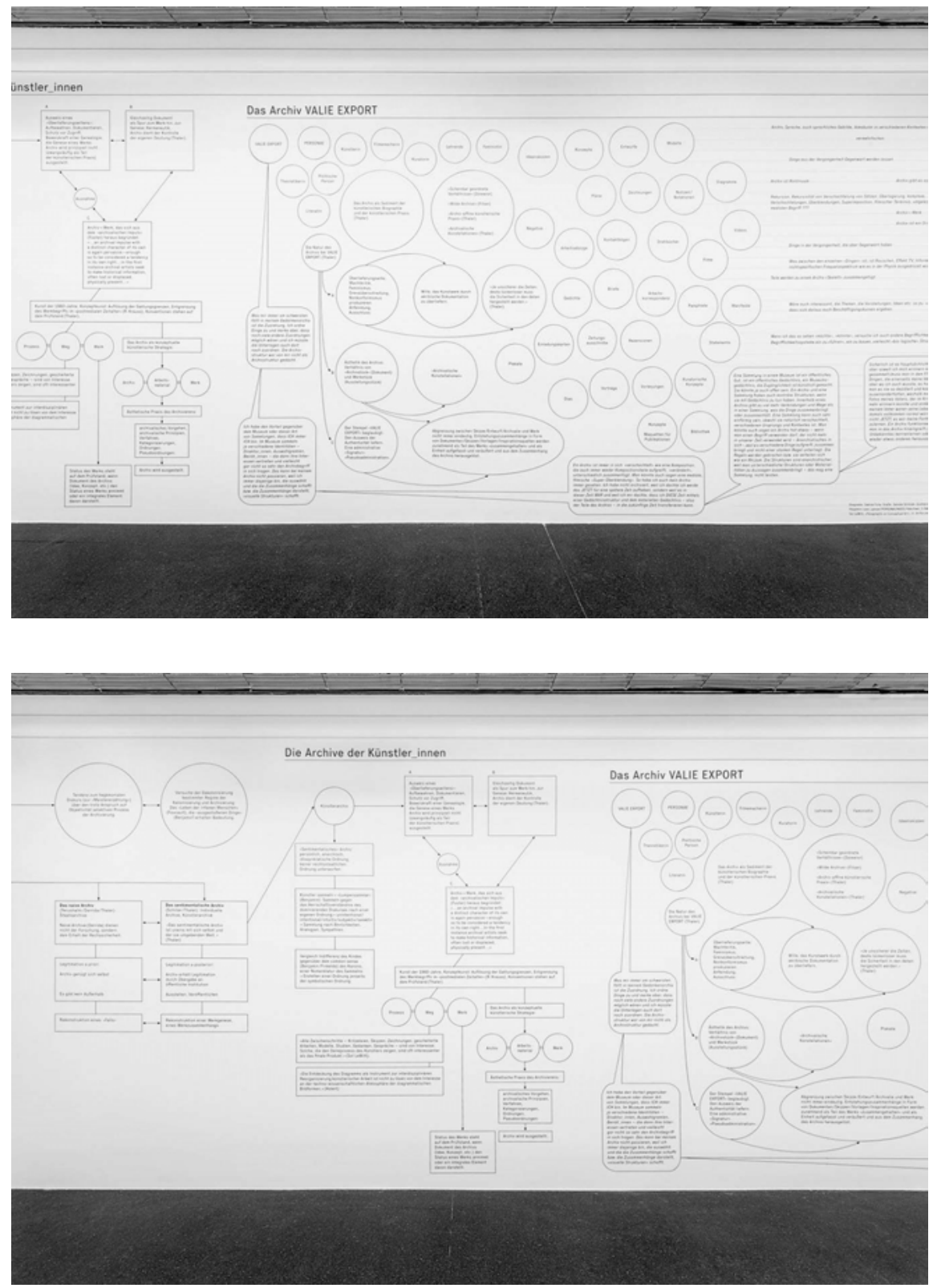

Abb. 1-4: VALIE EXPORT. Das Archiv als Ort künstlerischer Forschung, LENTOS Kunstmuseum Linz. 